\title{
STUDY ON KNOWLEDGE REASONING BASED ON EXTENDED FORMULAS
}

\author{
Chunyan Yang, Guanghua Wang, Yang Li, Wen Cai \\ Research Institute of Extension Engineering, Guangdong University of Technology, \\ Guangzhou, P.R. China 510090
}

Abstract: In artificial intelligence, knowledge representation and knowledge reasoning are very important problems. There are different reasoning rules for different knowledge representation methods. In this paper, extended formulas are established based on the extended analysis principle of basic-element, and the new knowledge representation methods and knowledge reasoning modes with extended formulas are put forward. Accordingly extended reasoning modes are established. The study will provide new formalized tools for knowledge mining and new reasoning technique for solving contradiction problems.

Key words: extended formula, knowledge reasoning, basic-element, knowledge representation, problem solving

\section{INTRODUCTION}

Extenics studies the extensibility of things, the rules and methods for opening up things, and then uses them to solve contradiction problems with formalized models. ${ }^{[1]}$ Its study objects are contradiction problems in the real world and its logic cells are basic-elements, including matter-element, affairelement and relation-element. Its logic base is extension logic. ${ }^{[2]}$ Its fundamental theory is extension theory, containing three pillars- basicelement theory, extension set theory and extension logic.

Extenics has a special method of its own called extension method, containing extension analysis method, extension transformation method, conjugate analysis method and extension set method. The technology applying extension theory and extension method to different special fields is 
called extension engineering ${ }^{[3]}$. Extenics has been in great progress, which has been made in both basic theory and application research. Not long after the birth of extension theory, many experts point out that "extension theory has very strong relation with $\mathrm{Al}^{[4]}$ and "it must penetrate into $\mathrm{AI}$ and its related subjects". ${ }^{[5]} \mathrm{AI}$ and Extenics are interrelated, which can be seen from their development process.

In AI, knowledge representation and knowledge reasoning are very important problems. There are different reasoning rules for different knowledge representation methods ${ }^{[6]}$. In this paper, extended formulas are established based on the extended analysis principle of basic-element, and the new knowledge representation methods and knowledge reasoning modes with extended formulas are put forward. Accordingly extended reasoning modes are established. The study will provide new formalized tools for knowledge mining and new reasoning technique for solving contradiction problems.

\section{BASIC-ELEMENTS AND KNOWLEDGE REPRESENTATION}

Basic-elements, including matter-element, affair-element and relationelement, are logic cells of Extenics. They can be used to express varied knowledge and get the form of knowledge representation standardization.

\subsection{The concepts of basic-elements}

The world is formed by all things. The reciprocity among things is called affairs. Things, affairs and their relations make up of the real world. In order to describe things, affairs and their relations with formalized methods, The conceptions of matter-element, affair-element and relation-element are provided.

Matter-element is a basic element to describe a matter. The ordered threedimensional group $R=(N, c, v)$ is called a matter-element. A matter' s name $N$, its characteristic $c$ and the measure $v$ about $c$ are three key elements of a matter- element.

A matter can have more than one characteristic. If we express a matter $N$ with its $\mathrm{n}$ characteristics $c_{1}, c_{2}, \cdots, c_{n}$ and the corresponding measures $v_{1}, v_{2}, \cdots, v_{n}$; the matter-element can be denoted by 


$$
R=\left[\begin{array}{c}
N, c_{1}, v_{1} \\
c_{2}, v_{2} \\
\cdots \cdots \\
c_{n}, v_{n}
\end{array}\right]=\left[\begin{array}{c}
R_{1} \\
R_{2} \\
\cdots \\
R_{n}
\end{array}\right]
$$

$R$ is called a n-dimensional matter-element, where $R_{i}=\left(N, c_{i}, v_{i}\right)$ is called $R$ 's component matter-element.

Affair-element is a basic element to describe an affair. The ordered three-dimensional group $I=(d, b, u)$ is called an affair-element. A verb' s name $d$, its characteristic $b$ and the measure $u$ about $b$ are three key elements of an affair-element.

Be similar to a n-dimensional matter-element, a n-dimensional affairelement can be denoted by

$$
I=\left[\begin{array}{c}
d, b_{1}, u_{1} \\
b_{2}, u_{2} \\
\cdots . . \\
b_{n}, u_{n}
\end{array}\right]=\left[\begin{array}{c}
I_{1} \\
I_{2} \\
\cdots \\
I_{n}
\end{array}\right]
$$

Relation-element is a basic element to describe a relation. The ordered three-dimensional group $Q=(s, a, w)$ is called a relation-element. A relation's name(or relation's symbol) $s$, its characteristic $a$ and the measure $w$ about $a$ are three key elements of a relation-element.

\subsection{The knowledge representation methods based on basic-element}

The traditional artificial intelligence(AI), based on symbol and logic, solves problems by knowledge representation and knowledge reasoning ${ }^{[6]}$. There are many different representation methods for the same problem, and they have different representation space. Whether the representations of a problem are good or not has great influence to the results and workload of solving the problem. Therefore, it will greatly improve the efficiency of problem solving in AI that properly selecting and correctly using knowledge representation methods.

There are many kinds of knowledge representation methods, such as predicate logic method, semantic network, frame representation, production rule and process representation, etc.. All of them have their own advantages and disadvantages ${ }^{[6]}$. Basic-elements, their operation formulas and compound-elements can give the representation methods a united form. That is to say, we can use basic-elements to represent predicate, production rule, semantic network and frame, etc ${ }^{[2]}$. Representing knowledge with basicelements, we can use transformation and reasoning to generate new 
And we call them combined formulas.

Given a matter-element $R=(N, c, v)$, if there exist matter-elements $R_{i}=\left(N_{i}, \quad c_{i}, \quad v_{i}\right),(i=1,2, \ldots, n)$, so that $R=R_{1} \oplus R_{2} \oplus \cdots \oplus R_{n}$, then

And we call it decomposition formula.

$$
R /\left\{R_{1}, R_{2}, \cdots, R_{n}\right\} \text {. }
$$

\section{EXTENDED REASONING MODES----THE KNOWLEDGE REASONING MODES BASED ON EXTENDED FORMULAS}

According to the above-mentioned extended formulas, we can get the following knowledge reasoning modes:

(1) divergent reasoning modes

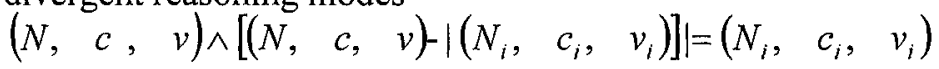

The formula means that $(N, c, v)-\left\{\left(N_{i}, c c_{i}, v_{i}\right)\right.$ are divergent formulas. If $(N, c, v)$ is a fact, then $\left(N_{i}, c_{i}, v_{i}\right)$ are facts. In the formula, $\left(N_{i}, c_{i}, v_{i}\right)$ can be the matter-elements with the same matter, the matterelements with the same characteristic, or the matter-elements with the same measure, etc..

(2) correlative reasoning modes

$$
\begin{aligned}
& (N, \quad c, v) \wedge\left[(N, c, v) \sim\left(N_{i}, \quad c_{i}, \quad v_{i}\right), v_{i}=f(v)\right] \\
& 1=\left[\left(N_{i}, \quad c_{i}, \quad v_{i}\right), v_{i}=f(v)\right]
\end{aligned}
$$

(3) implication reasoning modes

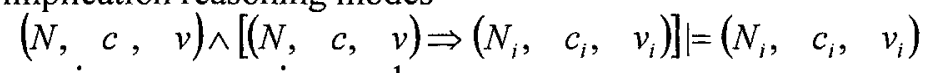

(4) opening-up reasoning modes

$$
\begin{aligned}
& (N, \quad c, v) \wedge\left(N_{i}, \quad c_{i}, \quad v_{i}\right) \wedge \\
& \left\{(N, \quad c, \quad v) \oplus\left(N_{i}, \quad c_{i}, \quad v_{i}\right)=\left[\begin{array}{lll}
N \oplus N_{i}, & c, & v \oplus c\left(N_{i}\right) \\
& c_{i}, & v_{i} \oplus c_{i}(N)
\end{array}\right]\right\} \\
& \mid=\left[\begin{array}{ccc}
N \oplus N_{i}, & c, & v \oplus c\left(N_{i}\right) \\
& c_{i}, & v_{i} \oplus c_{i}(N)
\end{array}\right]
\end{aligned}
$$

$$
\begin{aligned}
& (N, \quad c, v) \wedge\left[(N, \quad c, v) /\left\{\left(N_{1}, \quad c_{1}, \quad v_{1}\right), \cdots,\left(N_{n}, \quad c_{n}, \quad v_{n}\right)\right\}\right] \\
& \mid=\left\{\left(N_{1}, \quad c_{1}, v_{1}\right),\left(N_{2}, \quad c_{2}, v_{2}\right), \cdots,\left(N_{n}, \quad c_{n}, v_{n}\right)\right\}
\end{aligned}
$$


Taking advantage of the above knowledge reasoning modes, we can provide many kinds of formalized, operative thoughts and approaches for problems solving. It is especially more effective to contradiction problems solving.

\section{CASE STUDY-----SOLVING CONTRADICTION PROBLEMS WITH EXTENDED REASONING}

[A monkey picks bananas] A room has the height of $2.8 \mathrm{~m}$. At $1.8 \mathrm{~m}$ above the ground, a handful of bananas $B$ hang. The monkey $A$ wants to pick $B$. But when standing up, its body height is $0.5 \mathrm{~m}$, and its touching height is $0.7 \mathrm{~m}$. There are an estrade, which is $1.4 \mathrm{~m}$ high and has a weight of $15 \mathrm{~kg}$, a chair, $0.5 \mathrm{~m}$ high and a weight of $5 \mathrm{~kg}$, a cabinet, $1.8 \mathrm{~m}$ high and a weight of $50 \mathrm{~kg}$, and a table, $1.2 \mathrm{~m}$ high and a weight of $20 \mathrm{~kg}$. Suppose these objects are mobile(can be moved or be pushed), and the biggest impetus of $A$ is $25 \mathrm{~kg}$ (that it is, $A$ can push the objects under $25 \mathrm{~kg}$ weights).

Now we use the knowledge representation methods based on basicelement and extended reasoning to solve the contradiction problem. Therefore, we should formulate the problem and its core problem with formalized method. Suppose the goal is

$$
G=\left[\begin{array}{cc}
\text { obtain, subjection object, } & \text { bananas } B \\
\text { executive object, } & \text { monkey } A \\
\text { position, } & 1.8 m \text { height }
\end{array}\right]=\left[\begin{array}{ccc}
d, & b_{1}, & B \\
b_{2}, & A \\
b_{3}, & 1.8 m
\end{array}\right]
$$

the condition is

$$
L=(A \text {, height, } 0.5 \mathrm{~m})=\left(A, c_{0}, 0.5 \mathrm{~m}\right)
$$

the problem is denoted by $P=G^{*} L$.

Assume the appraisal characteristic $c_{0}=$ height. $c_{0 s}$, whose domain of value-measures is $X_{0}=<1.8,2.8>$, is the needed height for realizing the goal $G$. $c_{0 t}$ is the height which is provided by $z_{0}$ in condition $L$. In this case, $z_{0}=L$ $=\left(A, c_{0}, 0.5 \mathrm{~m}\right)$, then $c_{0 r}\left(z_{0}\right)=0.7 \mathrm{~m}$ (touching height).

$$
\text { Let } W=\left\{g \mid g=\left(z, c_{0 t}, c_{0 l}(z)\right)=\left(z, c_{0,}, x\right), z \in\left\{z_{0-p}, z_{0 \sim}, z_{0 \oplus}, z_{0 \Rightarrow}\right\}\right\} \text {, }
$$

then the core problem of the original problem $P$ is

$$
P_{0}=g_{0} * l_{0}=\left(z_{0}, c_{0 t}, c_{0 t}\left(z_{0}\right)\right) *\left(z, c_{0 s}, X_{0}\right) \text {. }
$$

According to the actual problem, we can establish the dependent function

$$
k(x)=\frac{x-1.8}{2.8-1.8}=x-1.8
$$

To $\forall g=\left(Z, c_{0 t}, x\right) \in W, Z=(N, c, c(N)), c=c_{0}$ or $c$ and $c_{0}$ is the characteristics with the same domain of measures, let 
then

$$
\widetilde{A}(T)=\left\{\left(g, y, y^{\prime}\right) \mid g \in T_{W} W, y=K(g)=k(x), y^{\prime}=T_{K} K\left(T_{g} g\right)\right\}
$$

$$
K_{0}(P)=K\left(g_{0}\right)=k\left[c_{0 t}\left(Z_{0}\right)\right]=\frac{0.7-1.8}{2.8-1.8}=-1.1<0
$$

So the problem $P$ is an incompatible problem. The reason is the value of $c_{0 t}$ (touching height) cannot fulfill the request of the problem.

Now we take advantage of the extended reasoning modes to solve this incompatible problem. To be simple, we only show the application of divergent reasoning mode and expansile reasoning mode.

(1) Making use of divergent reasoning modes

$$
\left(Z_{0}\right) \wedge\left(Z_{0}-1\left(A_{i}, c_{0}, c_{0}\left(A_{i}\right)\right)=Z_{i}\right) \mid=Z_{i},(i=1,2, \cdots, n)
$$

Let

$$
g_{i}=\left(Z_{i}, \quad c_{0 \mathrm{t}}, \quad c_{0 \mathrm{t}}\left(Z_{i}\right)\right) \triangleq\left(Z_{i}, \quad c_{0 \mathrm{t}}, x_{i}\right)
$$

According to the definition of dependent function, to make $y=K\left(g_{i}\right)>0$, we need $k\left(x_{i}\right)>0$, that is $k\left(c_{0 t}\left(Z_{i}\right)\right)=c_{0 t}\left(Z_{i}\right)-1.8>0$. Therefore, we can get $c_{0 t}\left(Z_{i}\right)>1.8$.

Then make a transformation,

$$
T_{1}=\left[\begin{array}{lll}
\text { replace }, & b_{1}, & A \\
& b_{2}, & A_{i}
\end{array}\right]
$$

That is, we can use a life-form $A_{i}$, whose touching height is over $1.8 \mathrm{~m}$ to replace $A$. So

$$
\begin{gathered}
T_{1} g_{0}=\left(T_{1 Z_{0}} Z_{0}, c_{0 t}, c_{0 t}\left(T_{1 Z_{0}} Z_{0}\right)\right)=\left(\left(A_{i}, c_{0}, c_{0}\left(A_{i}\right)\right), c_{0 t}, c_{0 t}\left(z_{i}\right)\right) \\
K\left(T_{1} g_{0}\right)=k\left(c_{0 t}\left(Z_{i}\right)\right)>0
\end{gathered}
$$

Now the incompatible problem is transformed to a compatible one.

(2) Making use of opening-up reasoning modes

Let

$$
\begin{gathered}
\left(Z_{0}\right) \wedge\left(Z_{i}\right) \wedge\left(Z_{0} \oplus Z_{i}=\left(A \oplus A_{i}, c_{0}, c_{0}(A) \oplus c_{0}\left(A_{i}\right)\right)=Z_{i}^{\prime}\right) \\
F Z_{i}^{\prime},(i=1,2, \cdots, n)
\end{gathered}
$$

$g_{i}{ }^{\prime}=\left(Z_{i}{ }^{\prime}, \quad c_{0 t}, \quad c_{0 t}\left(Z_{i}{ }^{\prime}\right)\right)=\left(Z_{i}{ }^{\prime}, c_{0 \mathrm{t}}, \quad 0.7 \oplus c_{0 \mathrm{t}}\left(A_{i}\right)\right) \triangleq\left(Z_{i}{ }^{\prime}, c_{0 \mathrm{t}}, x_{i}\right)$

According to the definition of dependent function, there is $k\left(x_{i}\right)>0$, that is

$$
0.7 \oplus c_{0 \mathrm{t}}\left(A_{i}\right)-1.8>0
$$

so that $c_{01}\left(A_{i}\right)>1.1$. That is to say, we can look for an object $A_{i}$, which is over $1.1 \mathrm{~m}$ high, to combine with monkey $A$ to solve the incompatible problem.

For example, we can use $Z_{1}=\left(\right.$ estrade $\left.A_{1}, c_{0}, 1.4 \mathrm{~m}\right), Z_{2}=\left(\operatorname{chair} A_{2}, c_{0}, 0.5 \mathrm{~m}\right)$, $Z_{3}=\left(\right.$ cabinet $\left.A_{3}, c_{0}, 1.8 \mathrm{~m}\right), Z_{4}=\left(\operatorname{table} A_{4}, c_{0}, 1.2 \mathrm{~m}\right), \cdots$ to combine with $A$.

So we can make transformation 


$$
T_{2}=\left[\begin{array}{ccc}
\text { add, } & b_{1}, & g_{0} \\
b_{2}, & g_{1} \\
b_{3}, & g_{0} \oplus g_{1} \\
b_{4}, & \text { push } A_{1} \\
b_{5}, & \text { manual } \\
b_{6}, & \text { beneath the bananas }
\end{array}\right]
$$

where the character $b_{4}$ expresses the mode of " $a d d$ ", $b_{5}$ expresses the tool and $b_{6}$ expresses the place. $T_{2}$ shows A pushes the table $A_{1}$ to the underside of the banana and stands on $A_{1}$, which is $1.4 \mathrm{~m}$ high. At this time,

$$
\begin{aligned}
T_{2} g_{0}=g_{0} \oplus g_{1}= & \left(\left(A, c_{0}, 0.5 \mathrm{~m}\right), c_{0 t}, 0.7 m\right) \oplus\left(\left(A_{1}, c_{0}, 1.4 \mathrm{~m}\right), c_{0}, 1.4 \mathrm{~m}\right) \\
= & \left(\left(A \oplus A_{1}, c_{0}, 1.9 \mathrm{~m}\right), c_{0 t}, 2.1 \mathrm{~m}\right) \\
& K\left(T_{2} g_{0}\right)=k(2.1)=2.1-1.8=0.3>0
\end{aligned}
$$

Now we have changed the incompatible problem to a compatible one.

Similarly, we can make transformations $T_{3}, T_{4}, T_{5}$, so that

$$
\begin{aligned}
& K\left(T_{3} g_{0}\right)=k(1.2)=1.2-1.8=-0.6<0 \\
& K\left(T_{4} g_{0}\right)=k(2.5)=2.5-1.8=0.7>0 \\
& K\left(T_{5} g_{0}\right)=k(1.9)=1.9-1.8=0.1>0
\end{aligned}
$$

Apparently, $T_{4}, T_{5}$ can change the incompatible problem to a compatible one, but $T_{3}$ cannot.

It can be seen from the above case that through establishing the extension model of contradiction problems and using extended reasoning mode and extension transformation, we can get many transformations, such as $T_{1}, T_{2}$, $T_{4}, T_{5}$, for solving contradiction problems. But their degrees of superiority are not same. We must do superiority evaluation according to the feasibility $\left(c_{1}\right), \operatorname{cost}\left(c_{2}\right)$ and $\operatorname{effect}\left(c_{3}\right)$, and then select the transformations with higher superiority degree to put in practice. In this case, we can know the superiority degree of $T_{2}, T_{5}$ are higher through simulating in computer and superiority evaluation calculation. Limited to space, they are omitted.

\section{CONCLUDING REMARKS}

The objective of knowledge representation and knowledge reasoning is problem solving ${ }^{[7]}$. In this paper, through the approach to knowledge reasoning modes based on extended formulas, we attempt to apply them to solve contradiction problems, and the case analysis has proved it is feasible. The study will provide new tools for knowledge representation and knowledge reasoning of $\mathrm{AI}$, and advance the study of knowledge reasoning. 
Moreover, it will probe new ways for intelligentizing the process of solving contradiction problems.

\section{ACKNOWLEDGEMENTS}

The research is supported by National Natural Science Foundation of China 70271060 and 70140003 and Guangdong Province Natural Science Foundation 010049.

\section{REFERENCES}

[1] W. Cai. Extension Theory and its application. Chinese Science Bulletin, Vol. 44, No. 17( 1999) 1538-1548.

[2] Cai Wen, Yang Chunyan, He Bin. Preliminary of extension logic[M]. Beijing: Science Press,2003(in Chinese).

[3] Cai Wen, Yang Chunyan and Lin Weichu. Methods of Extension Engineering. Beijing: Science Press, 2003

[4] P. Z. Wang. My Tentative Knowledge to Matter-element Analysis, From Matter-element Analysis to Extenics (Science and Technology Documentation Pub., Peking, 1995). (in Chinese)

[5] S. G. Hong. A New Study Orientation. From Matter-element Analysis to Extenics (Science and Technology Documentation Pub., Peking, 1995). (in Chinese)

[6] Cai Zixing, Xu Guangyou. Artificial intelligence and its application[M]. Beijing: Tingshua University Press,2004(in Chinese).

[7] George. F. Luger. Artificial intelligence-structures and strategies for complex problem solving[M]. Beijing: China Machine Press, 2003 